\title{
Duality between quantum and classical dynamics for integrable billiards
}

\author{
W. T. Lu, ${ }^{*}$ Weiqiao Zeng, and S. Sridhar ${ }^{\dagger}$ \\ Department of Physics and Electronic Materials Research Institute, \\ Northeastern University, Boston, Massachusetts 02115
}

(Dated: September 22, 2018)

\begin{abstract}
We establish a duality between the quantum wave vector spectrum and the eigenmodes of the classical Liouvillian dynamics for integrable billiards. Signatures of the classical eigenmodes appear as peaks in the correlation function of the quantum wave vector spectrum. A semiclassical derivation and numerical calculations are presented in support of the results. These classical eigenmodes can be observed in physical experiments through the auto-correlation of the transmission coefficient of waves in quantum billiards. Exact classical trace formulas of the resolvent are derived for the rectangle, equilateral triangle, and circle billiards. We also establish a correspondence between the classical periodic orbit length spectrum and the quantum spectrum for integrable polygonal billiards.
\end{abstract}

PACS numbers: 02.30.Ik, 03.65.-w, 05.20.Gg, 03.65.Sq

\section{INTRODUCTION}

Two sets of spectra can be associated with any dynamical system. The quantum spectrum, represented by the eigenvalues of the Schrödinger equation, is a set of real numbers for a closed system, while for an open system, the eigenvalues are complex and are called resonances. The classical dynamics can be described in terms of the spectrum of eigenvalues of the Liouville operator. For hyperbolic systems, the classical spectrum is comprised of the so-called Ruelle-Pollicott resonances [1,2] which determine the time evolution and relaxation of classical correlations.

Since the birth of quantum mechanics the correspondence between the classical and quantum properties has been examined from different perspectives. While the classical dynamics in the phase space is governed by the Liouville equation, the corresponding quantum dynamics in phase space is governed by the Moyal equation of the Wigner function. In the classical limit, the higher order terms of $\hbar$ in the Moyal equation vanish, and one retrieves the classical Liouvillian dynamics. This correspondence in the phase space properties has been studied by Brumer and collaborators [3]. In this paper, we obtain three principal results that establish a duality between quantum and classical dynamics in integrable billiards.

For billiard systems, it is natural to work in wave vector space $[4,5]$. We find that the two-level correlation of billiard systems in wave vector $k$ space is invariant with respect to the correlation interval. Peaks are observed in the quantum spectral correlations which are shown to be centered at the classical eigenmodes of the Liouvillian dynamics. Thus we establish a duality between the quantum wave vector spectrum $\left\{k_{n}\right\}$ and the classical eigenmodes $\left\{\gamma_{n}\right\}$ for integrable closed billiard systems,

\footnotetext{
*Electronic address: w.lu@neu.edu

${ }^{\dagger}$ Electronic address: s.sridhar@neu.edu
}

which can be expressed as

$$
C\left[f\left(\left\{k_{n}\right\}\right)\right]=g\left(\left\{\gamma_{n}\right\}\right) .
$$

Here $f\left(\left\{k_{n}\right\}\right)$ and $g\left(\left\{\gamma_{n}\right\}\right)$ are certain spectral functions with $C[f]$ an appropriately defined quantum correlation. The quantum wave vector spectral correlation directly leads to the classical eigenmodes. The results are numerically demonstrated for example systems including the two-dimensional rectangle, equilateral, and the circle billiards. A semiclassical derivation is provided supporting the results. Generalization to higher dimensional integrable systems is straightforward.

It is well known that the quantum spectrum can be calculated from classical periodic orbits (POs) through the Gutzwiller trace formula. Here we obtain an exact inverse result, viz., that the classical PO length spectrum can also be expressed in terms of the quantum wave vector spectrum, for integrable polygonal billiards. Finally, we also obtain exact classical trace formulas for integrable billiards.

In Sec. II, the quantum correlation is defined and the billiard systems under consideration are introduced. The corresponding classical dynamics are discussed, the classical eigenmodes and exact classical trace formula are obtained in Sec. III. A semiclassical derivation of the quantum correlation is provided in Sec. IV. Detailed numerical calculations supporting the main results are presented in Sec. V. Exact relation between the classical POs spectrum and the quantum spectrum is established for integrable billiards in Sec. VI. Finally, Sec. VII presents a summary and some remarks on the results.

\section{QUANTUM EIGENVALUES AND CORRELATIONS}

In this paper, we consider the wave vector density of states (DOS) as our quantum spectral function which is a sum of $\delta$-functions $\rho(k)=\sum_{n} \delta\left(k-k_{n}\right)$. In order to study the quantum spectral correlation, we use a Lorentzian- 
smoothed DOS

$$
\rho_{\epsilon}(k)=\frac{1}{\pi} \sum_{n} \frac{\epsilon}{\left(k-k_{n}\right)^{2}+\epsilon^{2}} .
$$

Here $\epsilon$ is a small width. In the limit $\epsilon \rightarrow 0$, one gets the stick spectrum. The continuous part of the DOS for two-dimensional closed billiard systems is obtained from the Weyl law as $\langle\rho(k)\rangle=(A / 2 \pi) k \pm L / 4 \pi$ with $A$ and $L$ the area and perimeter, respectively. The plus sign is for Neumann boundary condition (NBC) while minus sign for Dirichlet boundary condition (DBC). The fluctuation part of DOS is $\delta \rho_{\epsilon}(k)=\rho_{\epsilon}(k)-\langle\rho(k)\rangle$. Define the following correlation

$$
C_{\rho_{\epsilon}}(s) \equiv\left\langle\delta \rho_{\epsilon}(k) \delta \rho_{\epsilon}(k+s)\right\rangle_{k} .
$$

Here $\delta \rho_{\epsilon}(k)$ is with a window $\left[K_{0}-\Delta, K_{0}+\Delta\right]$ with certain $K_{0}$ and $\Delta$ such that $\Delta \gg \delta$, the mean level spacing. The range of average over $k$ is from $-\Delta-\min (0, s)$ to $\Delta-\max (0, s)$. The range of $s$ can be safely put as $-\Delta<s<\Delta$. The average over $k$ is defined as $\langle f(k)\rangle=(b-a)^{-1} \int_{a}^{b} f(k) d k$. Cross-correlation can be defined similarly for any two intervals $\left[K_{1}-\Delta, K_{1}+\Delta\right]$ and $\left[K_{2}-\Delta, K_{2}+\Delta\right]$ with arbitrary $K_{1}$ and $K_{2}$ [6].

We remark that the correlation $C_{\rho_{\epsilon}}(s)$ in Eq. (3) is essentially the two-level correlation $R_{2}(s)$. The difference is that here, there is no unfolding and no rescaling by the mean level spacing of the energy levels. For billiard systems, the correlation $C_{\rho_{\epsilon}}(s)$ in Eq. (3) is invariant with the choice of $K_{0}$ and $\Delta$ as long as one has enough quantum eigenvalues in the interval. For the Riemann zeros $[6-10]$, since the mean density is $\langle\rho(k)\rangle=(1 / 2 \pi) \ln (k / 2 \pi)$ which is very flat and practically a constant for large $k$, there is no real difference between unfolding and notunfolding. But for billiard systems, not-unfolding is essential to uncover the structures in the quantum spectral correlations.

Here we focus on the quantum spectra of three integrable systems, the rectangle, equilateral triangle, and circle billiards.

Rectangle billiard For rectangle billiard with sides $a$ and $b$, the eigen wave vector is

$$
k_{m n}=\pi \sqrt{(m / a)^{2}+(n / b)^{2}} .
$$

Here for DBC $m, n \geq 1$ and $m, n \geq 0$ for NBC.

Equilateral triangle billiard The equilateral triangle billiard is integrable only if all the sides have the same boundary condition. The eigenvalues are given by

$$
k_{m n}=(4 \pi / 3 a) \sqrt{m^{2}+n^{2}-m n}
$$

with $a$ the side length. For the equilateral triangle with DBC, $m \geq 2 n \geq 2$ while with NBC, $m \geq 2 n \geq 0$.

Circle billiard The circle billiard is integrable. The spectrum $\left\{k_{m n}\right\}$ is given by

$$
\begin{aligned}
& J_{m}\left(k_{m n} a\right)=0, \text { DBC, } \\
& J_{m}^{\prime}\left(k_{m n} a\right)=0, \text { NBC. }
\end{aligned}
$$

Here $a$ is the radius of the circle and $J_{m}(x)$ is the Bessel function of the first kind.

\section{CLASSICAL EIGENMODES AND TRACE FORMULA IN LIOUVILLIAN DYNAMICS}

We first discuss the classical eigenmodes of the Liouvillian dynamics. For Hamiltonian systems, the phase space density distribution $\varrho\left(q_{i}, p_{i}, t\right)$ is governed by the Liouville equation

$$
\frac{\partial}{\partial t} \varrho\left(q_{i}, p_{i}, t\right)=\hat{L} \varrho\left(q_{i}, p_{i}, t\right)
$$

where $\hat{L} \equiv\{H$,$\} is the Liouville operator. Since we are$ interested in the spectrum of $\hat{L}$ instead of the detailed solutions of the equations of motion, it is better to switch to the action-angle variables which are related to $q_{i}$ and $p_{i}$ through a canonical transformation [11]. In the actionangle variables, the Hamiltonian is a function of actions $I_{i}=(2 \pi)^{-1} \oint p_{i} d q_{i}, H=H\left(I_{i}\right)$. For fixed $I_{i}$, the phase space is a N-dimensional torus with area $(2 \pi)^{N}$ for a $\mathrm{N}$ dimensional integrable system. The equations of motion for the angle variables $\vartheta_{i}$ are $d \vartheta_{i} / d t=\omega_{i}$ with $\omega_{i} \equiv$ $\partial H / \partial I_{i}$, which give solutions as linear functions of time. These frequencies have dependence on action variables $I_{i}$ and are in general continuous. They are often associated with certain trajectories. Since the Hamiltonian is cyclic in $\vartheta_{i}$, the Liouville operator $\hat{L}$ assumes a much simpler form in terms of action-angle variables

$$
\hat{L}=-\sum_{i} \frac{\partial H}{\partial I_{i}} \frac{\partial}{\partial \vartheta_{i}}=-\sum_{j} \omega_{j} \frac{\partial}{\partial \vartheta_{j}} .
$$

The eigenvalues of operators $i \frac{\partial}{\partial \vartheta_{j}}$ all take integer values for motions on the torus. Thus $\omega_{i}$ are the primitive eigen frequencies of the Liouville operator $\hat{L}$ on the $N$ dimensional torus.

Except for one-dimensional systems, these frequencies $\omega_{i}$ are not the eigenmodes of the Liouville operator on the energy surface which has $2 N-1$ dimensions. In order to obtain the classical eigenmodes of the system, an ensemble average must be performed over the whole energy surface. A good way is to look at the trace of the classical evolution operator

$$
\operatorname{tr} e^{\hat{L} t}=\frac{1}{\mu} \sum_{\mathbf{n}} \int d \mu \exp (i \mathbf{n} \cdot \omega t) .
$$

Here $d \mu$ is the Liouville measure on the energy surface in phase space [12]. For two-dimensional integrable systems, one has

$$
d \mu=4 \pi^{2} \delta\left(E-H\left(I_{1}, I_{2}\right)\right) d I_{1} d I_{2} .
$$

Here the factor $4 \pi^{2}$ is from the integration over the two angle variables since any integrand we will consider for an integrable system is independent of the angle variables. The total measure is $\mu=4 \pi^{2} \iint d I_{1} d I_{2} \delta(E-H)=$ $d V / d E$ with $V$ the phase space volume. The Laplace transform will give the trace of the resolvent

$$
g(z) \equiv \operatorname{tr}(z-\hat{L})^{-1}=\int_{0}^{\infty} \operatorname{tr} e^{\hat{L} t} e^{-z l} d l .
$$


Here for billiards, the particle speed $v$ is set to be unity, thus time $t$ is identified with length $l$ throughout the paper. The classical eigenmodes will show up as singular points in the trace of the resolvent. For hyperbolic systems, the trace of the resolvent has simple poles which are called the Ruelle-Pollicott resonances $g(z)=\sum_{n} d_{n} /\left(z-\gamma_{n}\right)$ with $d_{n}$ the degeneracy. For generic systems, the classical trace $g(z)$ will have various kinds of singularities which can be associated with the classical eigenmodes.

Rectangle billiard For a particle with mass $\mathfrak{m}$ moving inside a rectangle billiard, the two actions are $I_{x}=$ $p a \cos \alpha / \pi$ and $I_{y}=p b \sin \alpha / \pi$ with $p=\sqrt{2 \mathfrak{m} E}$ and $\alpha$ the angle of the velocity with respect to the $x$-axis. One thus gets $\omega_{x}=(\pi v / a) \cos \alpha$ and $\omega_{y}=(\pi v / b) \sin \alpha$ with $v$ the particle speed in the billiard. Since the Jacobian determinant $\left|\partial\left(I_{x}, I_{y}\right) / \partial(p, \alpha)\right|$ is $p a b / \pi^{2}$, so one has $d \mu=4 a b \delta\left(E-p^{2} / 2 \mathfrak{m}\right) p d p d \alpha$. For the rectangle billiard, the two actions should be all positive, so $\alpha \in[0, \pi / 2]$ and the total measure is $\mu=2 \pi \mathfrak{m} a b$. One obtains

$$
\begin{aligned}
\operatorname{tr} e^{\hat{L} t} & =\frac{2}{\pi} \sum_{m, n=-\infty}^{\infty} \int_{0}^{\pi / 2} e^{i\left(m \omega_{x}+n \omega_{y}\right) t} d \alpha \\
& =\sum_{m, n=-\infty}^{\infty} J_{0}\left(k_{m n} l\right) .
\end{aligned}
$$

Here $k_{m n}$ is given by Eq. (4). This result was also obtained by Biswas [13]. The Laplace transform of the Bessel function $J_{0}(x)$ gives

$$
g_{\mathrm{rec}}(z)=\sum_{m, n=-\infty}^{\infty} \frac{1}{\sqrt{z^{2}+k_{m n}^{2}}} .
$$

The classical eigenmodes are given by $\gamma_{m n}=i k_{m n}$. These classical eigenmodes are discrete. The above trace formula of $g_{\mathrm{rec}}(z)$ diverges as $2 a b k_{N} / \pi$ with $k_{N}$ the cutoff of $k_{m n}$.

Equilateral triangle billiard For a particle in an equilateral triangle billiard, the classical dynamics is integrable. The phase space surface is a regular hexagon with parallel sides identified, and thus has the topology of a torus [14]. The classical eigenmodes are given by $\gamma_{m n}=i k_{m n}$ with $k_{m n}$ given by Eq. (5) and $m, n=0, \pm 1, \pm 2, \cdots$. The trace of the resolvent is

$$
g_{\mathrm{tri}}(z)=\frac{1}{3} \sum_{m, n=-\infty}^{\infty} \frac{1}{\sqrt{z^{2}+k_{m n}^{2}}} .
$$

The trace of the resolvent can be derived from the following expression

$$
\begin{aligned}
\operatorname{tr} e^{\hat{L} t} & =\sum_{m, n=-\infty}^{\infty} \int_{0}^{\pi / 3} e^{i(4 \pi / 3 a)[m \cos \alpha+n \cos (\pi / 3-\alpha)] l} \frac{d \alpha}{\pi} \\
& =\frac{1}{3} \sum_{m, n=-\infty}^{\infty} J_{0}\left(k_{m n} l\right)
\end{aligned}
$$

Though this is similar to that for the rectangle billiard, we are unable to give a derivation nor a physical interpretation for this expression.

Circle billiard For a particle in a circle billiard of radius $a$, the Hamiltonian is $H=p_{r}^{2} / 2 \mathfrak{m}+p_{\theta}^{2} / 2 \mathfrak{m} r^{2}$. The actions for the angular and radial freedoms are $I_{\theta}=p_{\theta}$ and $I_{r}=$ $\pi^{-1}\left(p^{2} a^{2}-I_{\theta}^{2}\right)^{1 / 2}-\pi^{-1} I_{\theta} \arccos \left(I_{\theta} / p a\right)$ with $p=\sqrt{2 \mathfrak{m} E}$ [15]. We parameterize the two actions as $I_{\theta}=p a \cos \alpha$ and $I_{r}=p a(\sin \alpha-\alpha \cos \alpha) / \pi$ with $0 \leq \alpha \leq \pi$ since $I_{r}$ is positive and $I_{\theta}$ can take positive or negative value [12]. The frequencies are $\omega_{r}=\pi v / a \sin \alpha$ and $\omega_{\theta}=\alpha v / a \sin \alpha$. They are both positive as required. Since the Jacobian determinant is $\left|\partial\left(I_{\theta}, I_{r}\right) / \partial(p, \alpha)\right|=\frac{1}{\pi} p a^{2} \sin ^{2} \alpha$, so $d \mu=$ $4 \pi a^{2} \delta\left(E-p^{2} / 2 \mathfrak{m}\right) p \sin ^{2} \alpha d p d \alpha$ and $\mu=2 \pi^{2} \mathfrak{m} a^{2}$. Thus one has

$$
\operatorname{tr} e^{\hat{L} t}=\frac{2}{\pi} \sum_{m, n=-\infty}^{\infty} \int_{0}^{\pi} e^{i(m \alpha+n \pi) l / a \sin \alpha} \sin ^{2} \alpha d \alpha
$$

and

$$
g_{\mathrm{cir}}(z)=\sum_{m, n=-\infty}^{\infty} \Xi(z ; m, n)
$$

with

$$
\Xi(z ; m, n) \equiv \frac{2}{\pi} \int_{0}^{\pi} \frac{a z \sin ^{4} \alpha d \alpha}{a^{2} z^{2} \sin ^{2} \alpha+(m \alpha+n \pi)^{2}} .
$$

Here $z=\gamma-i s$ with $\gamma>0$.

Simple expressions can be obtained for $\Xi(z ; m, n)$ with $m=0$. One has $\Xi(z ; 0,0)=1 / a z$. For $n \neq 0$, one has $\Xi(z ; 0, n)=\Xi(z / n ; 0,1) / n$ with

$$
\Xi(z ; 0,1)=\frac{2 \pi+\sqrt{a^{2} z^{2}+\pi^{2}}}{\left[\pi+\sqrt{a^{2} z^{2}+\pi^{2}}\right]^{2}} \frac{a z}{\sqrt{a^{2} z^{2}+\pi^{2}}} .
$$

Here we have used the following integral

$$
\int_{0}^{\pi} \frac{\cos 2 n \alpha d \alpha}{1+z^{2} \sin ^{2} \alpha}=\frac{\pi}{\sqrt{1+z^{2}}}\left(\frac{z}{1+\sqrt{1+z^{2}}}\right)^{2 n} .
$$

From the above expressions of $\Xi(z ; 0, n)$, one gets the classical eigenmodes

$$
\gamma_{0 n}=i n \pi / a, \quad n \geq 0 .
$$

For $m \neq 0$, no simple expression is found for $\Xi(z ; m, n)$, though the classical eigenmodes can still be obtained from its integral representation. For $m, n \neq 0$, the denominator in the integral representation of $\Xi(z ; m, n)$ may have two roots for certain $z$ being purely imaginary and $\alpha \in(0, \pi)$. If these two roots are equal, $\Xi(z ; m, n)$ will diverge. That is, $z= \pm i f\left(\alpha_{0}\right)$ with $f(\alpha)=(m \alpha+n \pi) / a \sin \alpha$ and its first derivative $f^{\prime}(\alpha)=$ $[m-(m \alpha+n \pi) \cot \alpha] / a \sin \alpha$ vanishes at $\alpha_{0}$. The classical eigenmodes are thus given by

$$
\gamma_{m n}=i m \sqrt{1+u^{2}} / a
$$




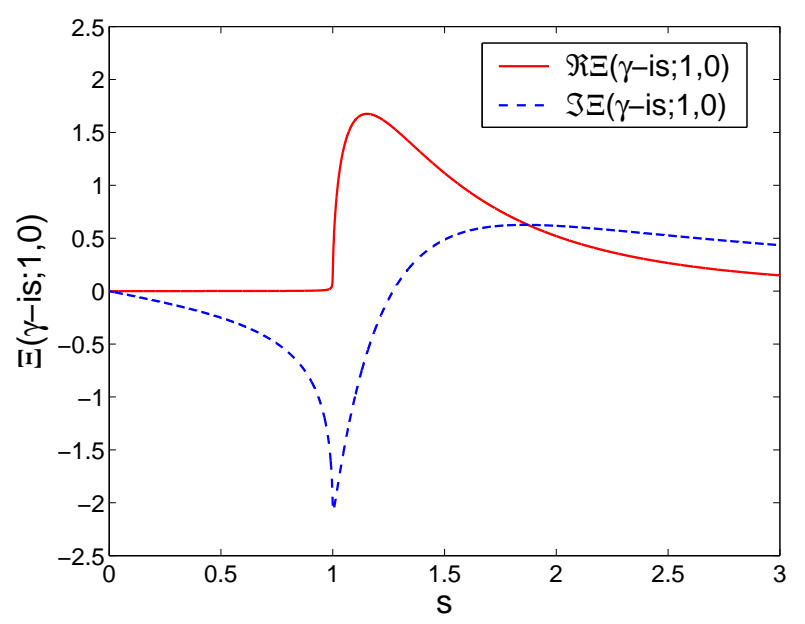

FIG. 1: Function $\Xi(\gamma-i s ; 1,0)$ with $\gamma=0.001$ and $a=1$. The solid line is real part $\Re \Xi(\gamma-i s ; 1,0)$ and the dashed line is the imaginary part $\Im \Xi(\gamma-i s ; 1,0)$.

with $u$ the solution of the transcendental equation

$$
u=n \pi / m+\arctan u .
$$

For $m \neq 0$ and $n=0$, one has $\Xi(z ; m, 0)=$ $\Xi(z / m ; 1,0) / m . \Xi(\gamma-i s ; 1,0)$ is free of divergence even when $\gamma \rightarrow 0$ and $\Re \Xi(-i s ; 1,0)=0$ for $s \leq 1$. We use $\Re$ for the real part and $\Im$ for the imaginary part throughout the paper. For $s>1, \Re \Xi(-i s ; 1,0)>0$ and has a maxmum at $s \simeq 1.1525$. The function $\Xi(\gamma-i s ; 1,0)$ with a small $\gamma$ is evaluated numerically and plotted in Fig. 1 . The resonance-looking hump in $\Re \Xi(-i s ; 1,0)$ gives rise to continuous classical eigenmodes and may be the reason for the apparent nonzero Lyapunov exponent [16] in the circle billiard and exponential short-time decay in circle billiard with small holes [17]. We emphasize that these classical eigenmodes bear no resemblance to the quantum eigenmodes. The first few discrete classical eigenmodes are listed in Table I.

TABLE I: A few low-lying quantum and classical eigenmodes of the circle billiard.

\begin{tabular}{c|c|c}
\hline \hline$k_{m n}$ for NBC & $\Im \gamma_{m n}$ & $k_{m n}$ for DBC \\
\hline 0 & 0 & 2.404825558 \\
\hline 1.841183781 & 3.141592654 & 3.831705970 \\
\hline 3.054236928 & 4.603338849 & 5.135622302 \\
\hline 3.831705970 & 5.943387741 & 5.520078110 \\
\hline 4.201188941 & 6.283185307 & 6.380161896 \\
\hline 5.317553126 & 7.221364982 & 7.015586670 \\
\hline 5.331442774 & 7.789705768 & 7.588342435 \\
\hline 6.415616376 & 8.460248568 & 8.417244140 \\
\hline 6.706133194 & 9.206677698 & 8.653727913 \\
\hline 7.015586670 & 9.424777961 & 8.771483816 \\
\hline 7.501266145 & 9.671732536 & 9.761023130 \\
\hline 8.015236598 & 10.566779006 & 9.936109524 \\
\hline \hline
\end{tabular}

For generic systems, the frequencies $\omega_{i}$ can not be unentangled with each other since the phase space is not a torus. Eq. (9) is not useful to obtain the classical eigenmodes. However the trace of the resolvent can be expressed in terms of POs which are the periodic solutions of the classical equations of motion. For hyperbolic systems, Cvitanović and Eckhardt [18] obtained the classical trace formula for the resolvent which is similar to the Gutzwiller semiclassical trace formula [19]. The classical trace formula for polygonal billiards is derived recently by Biswas [13]. Nevertheless, exact classical trace formulas can be derived directly from Eq. (9) for integrable billiards.

For the rectangle billiard, the fluctuation part of the classical trace formula can be directly derived from Eq. (12) as shown in the Appendix as

$$
\begin{aligned}
\delta g_{\mathrm{rec}}(z)= & \frac{2 a b}{\pi}\left[4 \sum_{m, n=1}^{\infty} \frac{e^{-z L_{m n}}}{L_{m n}}\right. \\
& \left.+2 \sum_{m=1}^{\infty}\left(\frac{e^{-z L_{m 0}}}{L_{m 0}}+\frac{e^{-z L_{0 m}}}{L_{0 m}}\right)\right] .
\end{aligned}
$$

Here the length of the POs is given by

$$
L_{m n}=2 \sqrt{(m a)^{2}+(n b)^{2}} .
$$

For the equilateral triangle billiard, along the same lines of derivation for the rectangle billiard, one gets

$$
\delta g_{\text {tri }}(z)=\frac{\sqrt{3} a^{2}}{2 \pi}\left(6 \sum_{m, n=1}^{\infty} \frac{e^{-z L_{m n}}}{L_{m n}}+6 \sum_{m=1}^{\infty} \frac{e^{-z L_{m 0}}}{L_{m 0}}\right)
$$

with

$$
L_{m n}=a \sqrt{3\left(m^{2}+n^{2}+m n\right)} .
$$

For the circle billiard, the classical trace formula can be derived directly from Eq. (16) as shown in the Appendix as

$$
\delta g_{\text {cir }}(z)=8 a^{2} \sum_{n=1}^{\infty} \sum_{m=2 n}^{\infty} f_{m n} \frac{\sin ^{4}(n \pi / m)}{L_{m n}} e^{-z L_{m n}}
$$

with

$$
\begin{aligned}
f_{m n} & =2 \text { for } m>2 n, \\
& =1 \text { for } m=2 n, \\
L_{m n} & =2 m a \sin (n \pi / m) .
\end{aligned}
$$

In summary the above represents exact trace formulas for the classical dynamics of integrable billiards.

\section{SEMICLASSICAL DERIVATION OF QUANTUM CORRELATIONS}

A semiclassical consideration can lead to the understanding of the quantum correlation. For large $k$, the semiclassical DOS for integrable polygonal billiards is [20]

$$
\delta \rho_{\epsilon}(k) \simeq \sqrt{\frac{k}{2 \pi^{3}}} \Re \sum_{p} a_{p} e^{(i k-\varepsilon) L_{p}-i \pi / 4} / \sqrt{L_{p}}
$$


The auto-correlation $C_{\rho_{\epsilon}}(s)$ can be written as the sum of diagonal and off-diagonal terms, $C_{\rho_{\epsilon}}(s)=C_{\text {diag }}^{\epsilon}(s)+$ $C_{\text {off }}^{\epsilon}(s)$. For integrable systems, the actions are uncorrelated since there is a direct relation between actions and the quantum spectrum. This leads to the cancelation of the off-diagonal terms,

$$
C_{\rho_{\epsilon}}(s) \simeq \frac{K_{0}}{4 \pi^{3}} \Re \sum_{p} \frac{a_{p}^{2}}{L_{p}} e^{-(2 \epsilon-i s) L_{p}} .
$$

Note that the above summation is over POs with different $L_{p}$. Different POs with the same $L_{p}$ are grouped together. For the rectangle billiard with irrational aspect ratio, $a_{p}=4 a b$ for all $\mathrm{PO}$ families $L_{m n}$ with nonzero $m$ and $n$ except for $L_{m 0}$ and $L_{0 n}$. Thus

$$
C_{\rho_{\epsilon}}(s) \simeq \frac{\left\langle a_{p}\right\rangle K_{0}}{8 \pi^{2}} \Re \delta g_{\mathrm{rec}}(2 \epsilon-i s) .
$$

With normalization at $s=0, C_{\rho_{\epsilon}}(s)$ and $\Re \delta g(2 \varepsilon-i s)$ are almost identical with each other though the relative heights of their peaks may be slightly different for rectangle billiards with rational aspect ratio or the equilateral triangle billiard. This is due to the fact that $a_{p}$ is no longer the same for all POs with different length for these billiards. This gives rise to extra fine structure in $C_{\rho_{\epsilon}}(s)$. We point out that the appearance of fine structures in $C_{\rho_{\epsilon}}(s)$ is not an indication of discrete nature of the quantum spectrum as claimed in $[21,22]$, rather that $a_{p}$ is not uniform.

For the circle billiard, there is no exact trace formula for $\rho(k)$ due to the lack of analytic expression for the zeros of the Bessel functions. For the billiard with DBC, the fluctuation part of the semiclassical DOS is [20,23]

$$
\delta \rho_{\epsilon}(k) \simeq 4 a^{2} \sqrt{\frac{k}{2 \pi}} \Re \sum_{n=1}^{\infty} \sum_{m=2 n}^{\infty} f_{m n} \frac{\sin ^{2}(n \pi / m)}{L_{m n}^{1 / 2}} e^{i \Phi_{m n}}
$$

with $\Phi_{m n}=(k+i \epsilon) L_{m n}+\nu_{m}, \nu_{m}=(6 m-1) \pi / 4$, and $f_{m n}$ given in Eq. (28). This trace formula will give the WKB quantization $[15,20]$

$$
k_{0 n}=(n+3 / 4) \pi
$$

which approximates the zeros of $J_{0}(z)$ and

$$
k_{m n}=m \sqrt{1+u^{2}}
$$

with

$$
u=(n+3 / 4) \pi / m+\arctan u
$$

for the approximate zeros of $J_{m}(z)$ with $m>0$. For the billiard with $\mathrm{NBC}$, the factor $3 / 4$ should be replaced by $1 / 4$. With either boundary condition, the diagonal part of the auto-correlation is

$$
C_{\rho_{\epsilon}}(s) \simeq \frac{16 a^{4} K_{0}}{\pi} \Re \sum_{n=1}^{\infty} \sum_{m=2 n}^{\infty} \frac{\sin ^{4}(n \pi / m)}{L_{m n}} e^{-(2 \epsilon-i s) z L_{m n}} .
$$

For the circle billiard, one has

$$
C_{\text {diag }}^{\epsilon}(s) \simeq \frac{4 \pi a^{2} K_{0}}{8 \pi^{2}} \Re \delta g_{\text {cir }}(2 \epsilon-i s) .
$$

Thus the quantum correlation is determined by the classical eigenmodes.

\section{NUMERICAL CALCULATION OF QUANTUM CORRELATIONS}

Numerical calculations of $C_{\rho_{\epsilon}}(s)$ were carried out to confirm the results discussed here. Results for the rectangle billiard with DBC are shown in Fig. 2. For the equilateral triangle billiard of side length $a=1$ with $\mathrm{DBC}$, the spectral correlation is shown in Fig. 3. Since the spectrum of the rectangle and equilateral triangle billiards with NBC is almost the same as the counterparts with DBC, their spectral correlations are essentially the same. The spectral correlations for the circle billiard of unit radius is shown in Fig. 4. About $10^{6}$ quantum eigenvalues are used in the above calculations.

As seen from the above figures, the auto-correlations $C_{\rho_{\epsilon}}(s)$ are almost the same for different spectra segments, confirming the correlation invariance. It is quite obvious that the peaks of the quantum wave vector correlation are located at $s=\Im \gamma_{m n}$ with $\gamma_{m n}$ the classical eigenmodes.

In Fig. 2, the rectangle billiard has an irrational aspect ratio. In this case, almost all the $\mathrm{PO}$ families have the same summation weight. With fixed quantum spectral width $\epsilon$, for a finite segment of quantum spectrum, the difference between the correlation $C_{\rho_{\epsilon}}(s)$ and the classical trace $\Re \delta g(2 \epsilon-i s)$ can be viewed as just noise if both are normalized at $s=0$. This noise will be reduced and eventually disappears if either the number of the quantum eigenvalue in the quantum spectral segment or the width $\epsilon$ increase. However for the equilateral triangle billiard as shown in Fig. 3, the difference between $C_{\rho_{\epsilon}}(s)$ and $\Re \delta g(2 \epsilon-i s)$ will persist even if the center $K_{0}$ of the quantum spectral segment is pushed to infinity. Actually, there are five curves with different $K_{0}$ plotted in Fig. 3. They are hardly distinguishable from each other. The classical trace $\Re \delta g(2 \epsilon-i s)$ has no structure between classical eigenmodes. The presence of small peaks between classical eigenmodes in $C_{\rho_{\epsilon}}(s)$ is what we called fine structure in the previous section and is due to the nonuniformity of $a_{p}$.

An interesting point about the circle billiard is that there are some resonance-like humps in the correlation as shown in Fig. 4 between the origin and the first nonzero discrete classical eigenmodes $\gamma_{01}=i \pi$. The hump between $[1, \pi / 2]$ is very prominent. These humps may be the precursor of Ruelle-Pollicott resonances in chaotic systems such as the Bunimovich stadium.

Two comments are in order. The first is that though it is tempting to make a one-one correspondence between the quantum and the classical eigenmodes for integrable systems, actually this is not true. For any segment of 

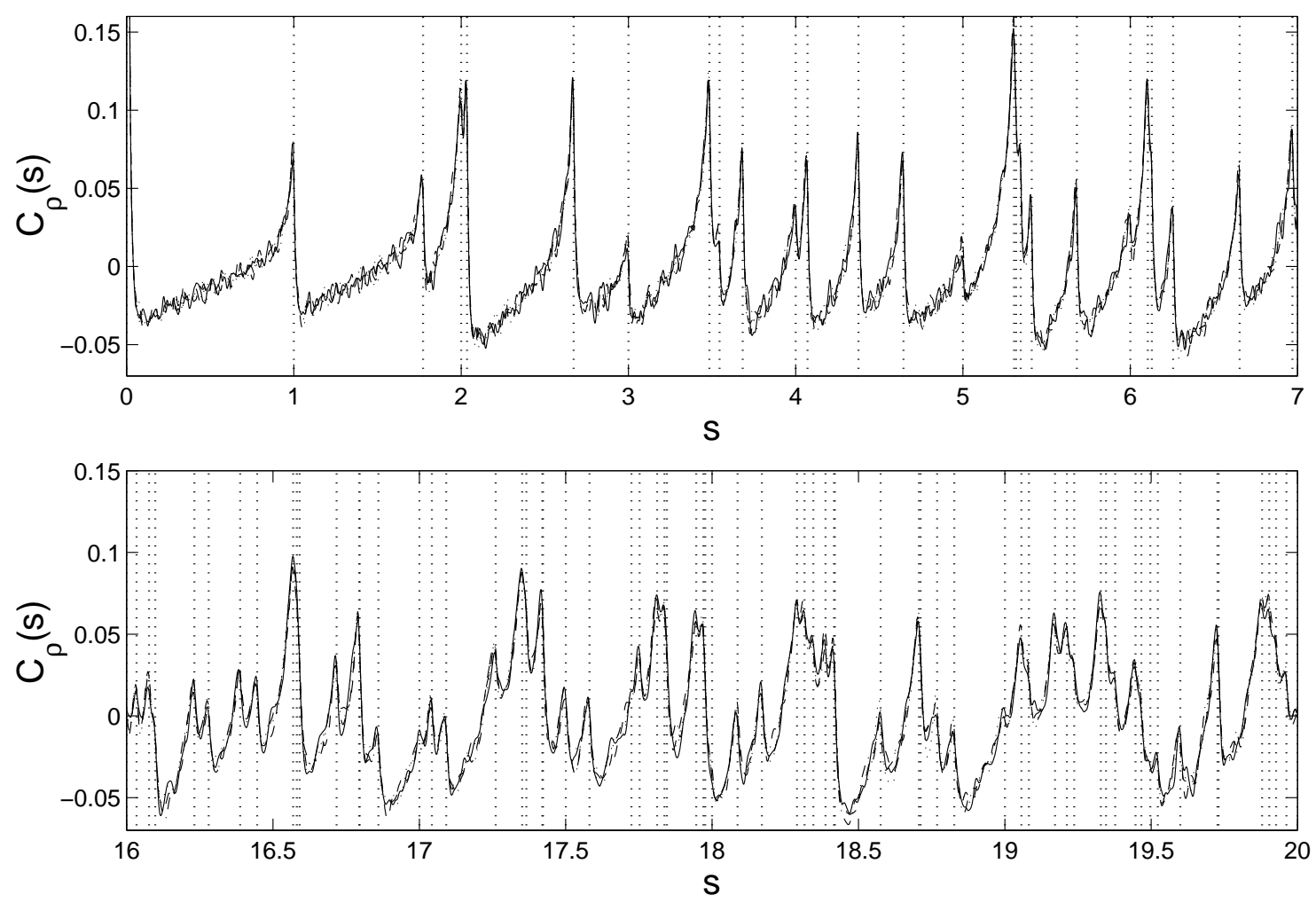

FIG. 2: Auto-correlation $C_{\rho_{\epsilon}}(s)$ of the rectangular billiard with sides $a=\pi$ and $b=\sqrt{\pi}$. Here $\epsilon=0.005$. $10^{6}$ quantum eigenmodes with $0<k_{m n}<1500$ are included in $\delta \rho_{\epsilon}(k)$. The auto-correlations $C_{\rho_{\epsilon}}(s)$ for $\delta \rho_{\epsilon}(k)$ in four equal intervals [0,375], $[375,750],[750,1125],[1125,1500]$ are calculated. They are normalized such that $C_{\rho_{\epsilon}}(0)=1$ and all collapse to the same curve. Vertical dashed lines indicate the location of the classical eigenmodes.

the quantum spectra, the auto-correlation will lead to the same low-lying classical eigenmodes as exemplified by the invariance of the quantum spectral correlations. The second is that in general, there is no correspondence between the trace of the classical project operator and the quantum Neumann spectrum. One can clearly see this for the circle billiard. For the rectangle and equilateral triangle billiards, the fact that the quantum NBC spectrum is also the classical eigenmodes is a coincident instead of a rule for polygonal billiard. One can view the circle billiard as the limit of equilateral polygon with increasing number of sides.

\section{CORRESPONDENCE BETWEEN CLASSICAL PERIODIC ORBIT LENGTH SPECTRUM AND QUANTUM WAVE VECTOR SPECTRUM}

In the previous sections, we established a duality between quantum and classical eigen spectra through quantum correlations. This duality was also established for hyperbolic systems [24]. We believe this can also be applied to generic systems.

On the other hand, the celebrated Gutzwiller trace formula indicates a direct duality between the quantum and classical dynamics such that the quantum DOS is expressed in terms of classical POs. As a complement to the Gutzwiller trace formula, the classical PO length spectrum can also be expressed in terms of quantum wave vector spectrum. This classical POs spectrum is crucial for the calculation of classical eigen spectrum using the Cvitanović-Eckhardt classical trace formula [18].

For integrable billiards, the classical trace formulas we derived in Sec. III assume the form

$$
\delta g(z)=\frac{2}{\pi} \sum_{p} \frac{a_{p}}{L_{p}} e^{-z L_{p}}
$$

with $a_{p}$ the area visited by the PO family with length $L_{p}$. This formula is also valid for generic polygonal billiard [13]. In order to obtain the classical eigenmodes, the number of $\mathrm{PO}$ families defined as $N(l)=\sum_{p} a_{p} \Theta\left(l-L_{p}\right)$ should be known. Here $\Theta(x)$ is the step function. $N(l)$ has the unit of billiard area. It was proved that the POs are dense for rational polygonal billiards [25, 26], $N(l) \propto l^{2}$. Recently Biswas obtained an expression of $N(l)$ in terms of the quantum spectrum with NBC [27]. Actually, $N(l)$ can also be expressed in terms of the quantum spectrum with DBC on the same basis. Here we give a different derivation of this connection and reveal the domain of its validity. Often the importance of isolated 

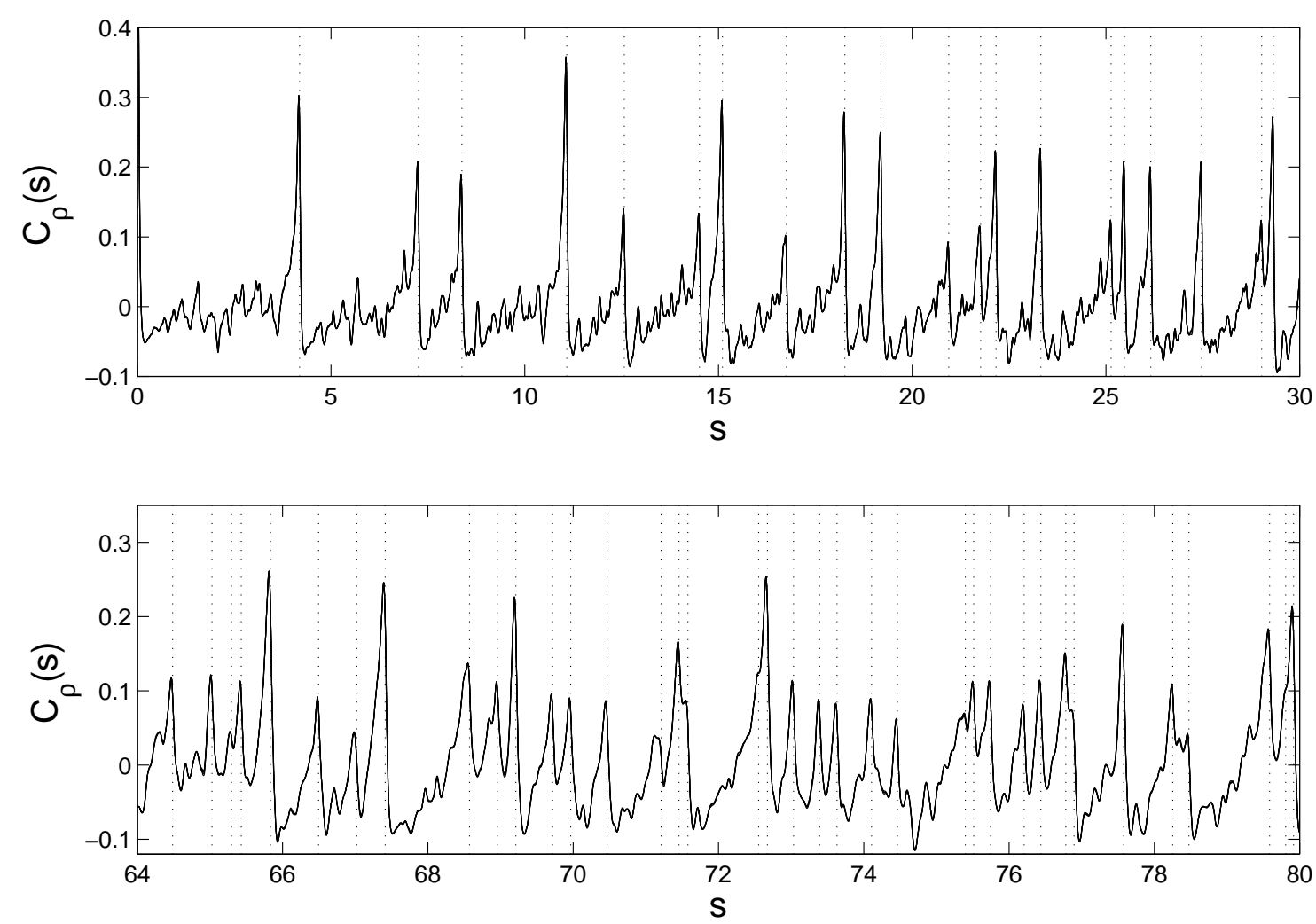

FIG. 3: Auto-correlation $C_{\rho_{\epsilon}}(s)$ of the equilateral triangle billiard with side length $a=1$. About $10^{6}$ quantum eigenmodes with $0<k_{m n}<5390$ are included in $\delta \rho_{\epsilon}(k)$ with $\epsilon=0.059$. The auto-correlations $C_{\rho_{\epsilon}}(s)$ for $\delta \rho_{\epsilon}(k)$ in five equally divided intervals are calculated, normalized at the origin, and plotted together. Vertical dashed lines indicate the location of the classical eigenmodes.

POs is neglected in this connection. We find that they are key to determine the proliferation of the PO families.

Since we focus on integrable billiards, diffractive POs and non-periodic orbits [28-30] are absent, the wave vector DOS of integrable polygonal billiards is

$$
\begin{aligned}
\rho(k)= & \langle\rho(k)\rangle+c_{0} \delta(k)+\frac{k}{2 \pi} \sum_{p} a_{p} J_{0}\left(k L_{p}\right) \\
& -\frac{1}{2 \pi} \sum_{p} b_{p} \cos k \ell_{p} .
\end{aligned}
$$

Here $c_{0}$ is the corner term and $b_{p}$ is the weight for isolated PO with length $\ell_{p}$ [20]. For two-dimensional billiards with DBC, the average DOS is $\langle\rho(k)\rangle=A k / 2 \pi-L / 4 \pi$. Since $\rho(k)=\sum_{n} \delta\left(k-k_{n}\right)$, multiplying both sides of Eq. (38) by $2 \pi l J_{1}(k l) / k$ and integrating over $k$ from zero to infinity, one gets

$$
\begin{aligned}
N(l)= & \frac{\pi}{4}\left(\alpha-2 c_{0}\right) l^{2}+\frac{L-\beta}{2} l-A+2 \pi l \sum_{n=1}^{\infty} \frac{1}{k_{n}} J_{1}\left(k_{n} l\right) \\
& +l \sum_{n=1}^{\infty} \sum_{p^{\prime}} \frac{b_{p^{\prime}}}{2 n} J_{1}\left(2 n \pi l / \ell_{p^{\prime}}\right) .
\end{aligned}
$$

The summation over $p^{\prime}$ is for the primitive isolated $\mathrm{PO}$ and

$$
\alpha=\sum_{p^{\prime}} \frac{b_{p^{\prime}}}{\ell_{p^{\prime}}}, \quad \beta=\sum_{p^{\prime}} b_{p^{\prime}}
$$

In the above derivation, use has been made of the following identities [31]

$$
\begin{aligned}
& \int_{0}^{\infty} d k J_{0}\left(k L_{p}\right) J_{1}(k l)=\frac{1}{l} \Theta\left(l-L_{p}\right), \\
& \int_{0}^{\infty} \frac{d k}{k} \cos \left(k \ell_{p}\right) J_{1}(k l)=\frac{1}{l} \Re \sqrt{l^{2}-\ell_{p}^{2}} \\
& \Re \sum_{n=1}^{[x]} \sqrt{x^{2}-n^{2}}=\frac{\pi}{4} x^{2}-\frac{1}{2} x+\frac{x}{2} \sum_{n=1}^{\infty} \frac{1}{n} J_{1}(2 n \pi x) .
\end{aligned}
$$

The terms $\pi \alpha l^{2} / 4$ and $\beta l / 2$ in $N(l)$ are from the first two terms of the third identity. Thus one has the average

$$
\langle N(l)\rangle=\frac{\pi}{4}\left(\alpha-2 c_{0}\right) l^{2}+\frac{L-\beta}{2} l-A .
$$

Hence the proliferation of $\mathrm{PO}$ families is determined by the isolated $\mathrm{PO}$ and the corner term in the quantum trace formula (38). This reveals an intimate connection between them. 

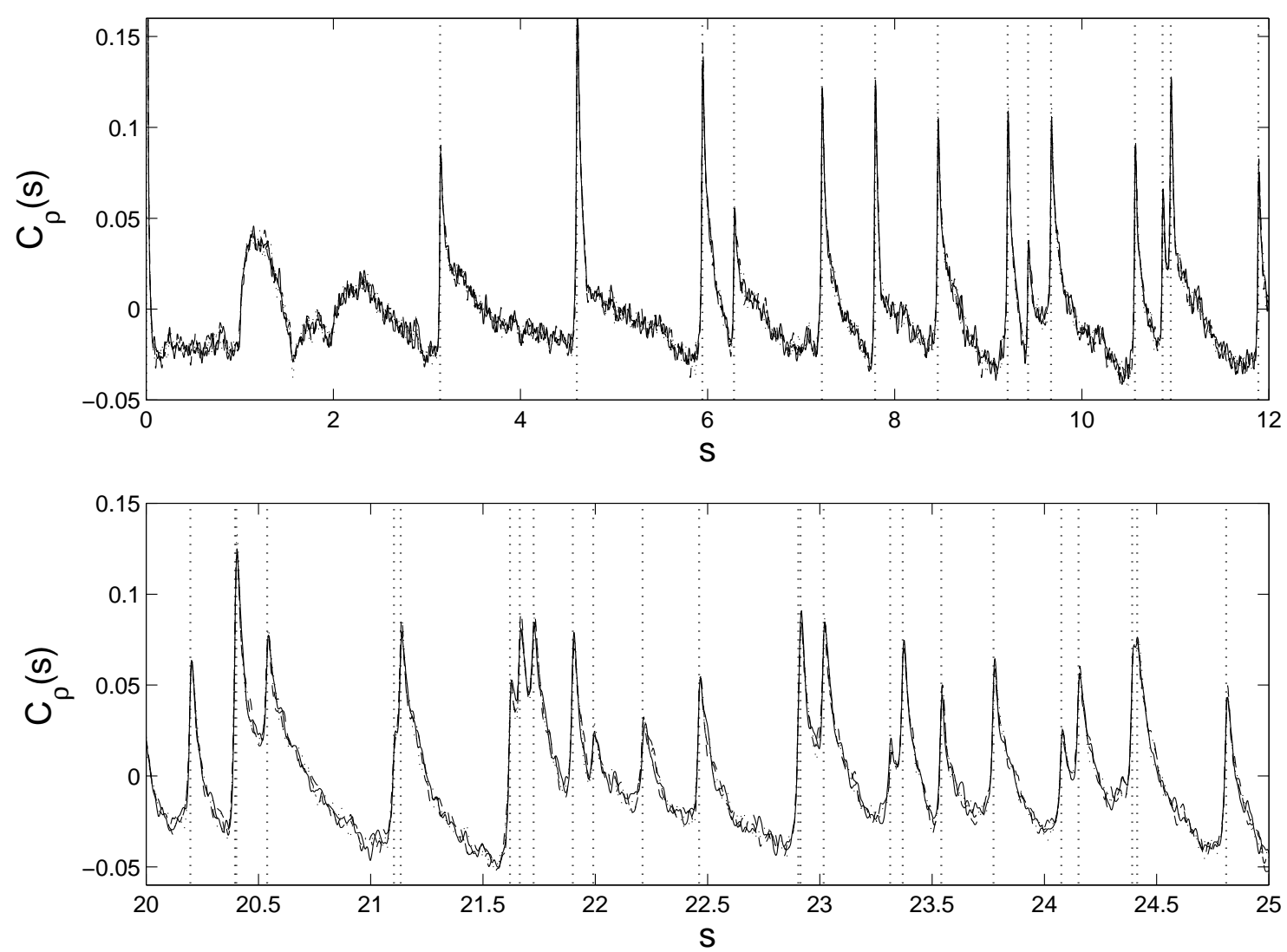

FIG. 4: Auto-correlation $C_{\rho_{\epsilon}}(s)$ of the circle billiard with radius $a=1$. About $2.3 \times 10^{6}$ quantum eigenmodes with $0<$ $k_{m n}<3000$ are included in $\delta \rho_{\epsilon}(k)$ with $\epsilon=0.005$. The auto-correlations $C_{\rho_{\epsilon}}(s)$ for $\delta \rho_{\epsilon}(k)$ in four equally divided intervals are calculated and normalized at the origin. Vertical dashed lines indicate the location of the classical eigenmodes. The two humps in the interval $[1,3]$ correspond to $\Re \Xi(2 \epsilon-i s ; 1,0)$ and $\Re \Xi(2 \epsilon-i s ; 2,0)$, respectively.

Exact expression of $N(l)$ can be obtained for the rectangle and equilateral triangle billiards. For the rectangle billiard with $\mathrm{DBC}$, the exact trace formula [20] will give $c_{0}=1 / 2, \alpha=2, \beta=L=2(a+b)$. One has exactly

$$
\begin{aligned}
N(l)= & \frac{\pi}{4} l^{2}-a b+2 \pi l \sum_{m, n=1}^{\infty} \frac{1}{k_{m n}} J_{1}\left(k_{m n} l\right) \\
& +l \sum_{n=1}^{\infty} \frac{1}{n}\left[a J_{1}(n \pi l / a)+b J_{1}(n \pi l / b)\right] .
\end{aligned}
$$

Thus $\langle N(l)\rangle=\pi l^{2} / 4-a b$. We stress that there is no linear term and the constant term is negative in $\langle N(l)\rangle$, contrary to the expression obtained by Jain [32]. As a comparison, the number of quantum states is

$$
\begin{aligned}
\mathcal{N}(k)= & \frac{a b}{4 \pi} k^{2}-\frac{a+b}{2 \pi} k+\frac{1}{4}+\frac{k}{2 \pi} \sum_{p} \frac{a_{p}}{L_{p}} J_{1}\left(k L_{p}\right) \\
& -\frac{1}{2 \pi} \sum_{n=1}^{\infty} \frac{1}{n}(\sin 2 n k a+\sin 2 n k b)
\end{aligned}
$$

which is obtained from Eq. (38) through an integration. Here the summation over $p$ is given by Eq. (23). One has $\langle\mathcal{N}(k)\rangle=a b k^{2} / 4 \pi-(a+b) k / 2 \pi+1 / 4$ as expected.
For the equilateral triangle billiard with DBC [20], one has $c_{0}=2 / 3, \alpha=2, \beta=L=3 a$. Thus

$$
\begin{aligned}
N(l)= & \frac{\pi}{6} l^{2}-\frac{\sqrt{3} a^{2}}{4}+2 \pi l \sum_{n=1}^{\infty} \frac{1}{k_{n}} J_{1}\left(k_{n} l\right) \\
& +\frac{3}{2} l \sum_{n=1}^{\infty} \frac{a}{n} J_{1}(4 n \pi l / 3 a)
\end{aligned}
$$

with $\langle N(l)\rangle=\pi l^{2} / 6-\sqrt{3} a^{2} / 4$. The number of quantum states is

$$
\begin{aligned}
\mathcal{N}(k)= & \frac{\sqrt{3} a^{2}}{16 \pi} k^{2}-\frac{3 a}{4 \pi} k+\frac{1}{3}+\frac{k}{2 \pi} \sum_{p} \frac{a_{p}}{L_{p}} J_{1}\left(k L_{p}\right) \\
& -\frac{1}{\pi} \sum_{n=1}^{\infty} \frac{1}{n} \sin (3 n k a / 2) .
\end{aligned}
$$

Here the summation over $p$ is given by Eq. (25). One has $\langle\mathcal{N}(k)\rangle=\sqrt{3} a^{2} k^{2} / 16 \pi-3 a k / 4 \pi+1 / 3$.

Similarly, one gets

$$
P(l) \equiv \sum_{p} \frac{a_{p}}{L_{p}} \delta\left(l-L_{p}\right)
$$




$$
\begin{aligned}
= & \frac{\pi}{2}\left(\alpha-2 c_{0}\right)-A k_{N} J_{1}\left(k_{N} l\right) / l+\frac{L-\beta}{2 l} \\
& +2 \pi \sum_{n=1}^{N} J_{0}\left(k_{n} l\right)+\pi \sum_{n=1} \sum_{p^{\prime}} \frac{b_{p^{\prime}}}{\ell_{p^{\prime}}} J_{0}\left(2 n \pi l / \ell_{p^{\prime}}\right), \\
S(l) \equiv & \sum_{p} a_{p} \delta\left(l-L_{p}\right) \\
= & \frac{\pi}{2}\left(\alpha-2 c_{0}\right) l-A k_{N} J_{1}\left(k_{N} l\right)+\frac{L-\beta}{2} \\
& +2 \pi l \sum_{n=1}^{N} J_{0}\left(k_{n} l\right)+\pi l \sum_{n=1} \sum_{p^{\prime}} \frac{b_{p^{\prime}}}{\ell_{p^{\prime}}} J_{0}\left(2 n \pi l / \ell_{p^{\prime}}\right) .
\end{aligned}
$$

Here $k_{N}$ is a large cutoff in the quantum spectrum. Exact expressions of $P(l)$ and $S(l)$ can be obtained for the rectangle and equilateral triangle billiards.

For billiard with uniform boundary condition, $a_{p}$ in the quantum trace formula (38) are all positive, the quantity $P(l)$ is just the classical quantity $(\pi / 2) \operatorname{tr} \exp (-\hat{L} t)$. The Laplace transform of $P(l)$ will give the fluctuation part of the classical trace $g(z)$

$$
\begin{aligned}
\delta g(z)= & \left(\alpha-2 c_{0}\right) / z-\frac{2 A}{\pi}\left(\sqrt{z^{2}+k_{N}^{2}}-z\right) \\
& +\sum_{n=1}^{N} \frac{4}{\sqrt{z^{2}+k_{n}^{2}}}+\sum_{n=1} \sum_{p^{\prime}} \frac{2 b_{p^{\prime}} / \ell_{p^{\prime}}}{\sqrt{z^{2}+\left(2 n \pi / \ell_{p^{\prime}}\right)^{2}}} .
\end{aligned}
$$

Here we ignored the term $(L-\beta) / 2 l$ in $P(l)$ since it vanishes for integrable polygonal billiards and made use of the Laplace transforms of Bessel functions

$$
\begin{aligned}
\int_{0}^{\infty} l^{-1} J_{1}\left(k_{N} l\right) e^{-z l} d l & =\frac{1}{k_{N}}\left(\sqrt{z^{2}+k_{N}^{2}}-z\right), \\
\int_{0}^{\infty} J_{0}\left(k_{n} l\right) e^{-z l} d l & =\frac{1}{\sqrt{z^{2}+k_{n}^{2}}} .
\end{aligned}
$$

Thus the classical eigenmodes are $\gamma_{n}=i k_{n}$ and $\gamma_{n}=$ $i 2 n \pi / \ell_{p^{\prime}}$ with $\ell_{p^{\prime}}$ the primitive isolated PO without repetition.

The results for $N(l), P(l), S(l)$ establish a correspondence between the classical PO length spectrum and the quantum spectrum for integrable polygonal billiards.

For the circle billiard, no exact expression of $N(l)$ can be obtained since we don't have an exact quantum trace formula. Nevertheless, an approximation of $N(l)$ could be derived similarly through the semiclassical trace formula.

We remark that the above equations may be applicable to non-integrable billiards only if the contribution to the quantum trace formula from diffractive POs and nonperiodic orbits can be safely ignored. This is not the case for generic polygonal billiards. For example for the $\pi / 3$ rhombus billiard, diffractive POs are nearly as dense as the PO families. The classical spectrum is thus different from the quantum spectrum though they may partially overlap with each other.

\section{CONCLUSIONS AND REMARKS}

In this paper, we directly obtained the classical eigenmodes of the Liouvillian dynamics and exact classical trace formula of the resolvent for integrable closed systems including the rectangle, equilateral triangle and the circle billiards, and showed that the peaks in the quantum wave vector spectral correlation coincide with the classical eigenmodes. We also established a correspondence between the classical PO length spectrum and the quantum wave vector spectrum for polygonal integrable billiards.

We have shown earlier in open chaotic systems, specifically the $n$-disk billiards, that the auto-correlation of the resonant quantum spectrum carries the fingerprints of the classical resonances, also known as the RuellePollicott resonances. For the Riemann zeros which are the quantum eigenmodes on negative constant curvature, the spectral correlation also leads to the "classical" eigenmodes $[6,10]$. We speculate that this also holds for chaotic and generic billiard systems. A direct duality between classical POs spectra and quantum spectra is also obtained for integrable billiards.

While for a quantum system, there are many ways to solve the Schrödinger equation, the classical spectrum is very difficult to get at. Direct numerical simulation of tracing classical trajectories is of limited use due to the presence of the Lyapunov exponent and computer round off error. The trace formula in terms of classical POs provide another correspondence between the quantum and classical dynamics. The usefulness of these trace formula to obtain classical [18] and quantum spectra [19] is marred by the divergence due to the exponential proliferation of POs. Though the classical eigenmodes are difficult to calculate for generic systems, our approach provides a simple way to obtain them.

Our results can be readily tested in wave-mechanical experiments, such as the microwave analogs of quantum billiards [33]. There the measured transmission is $T(k)=\sum_{n} c_{n} /\left[\left(k-k_{n}\right)^{2}+\epsilon_{n}^{2}\right]$. Earlier work has shown in $n$-disk open systems that the peaks of the correlation $C_{T}(s) \equiv\langle T(k) T(k+s)\rangle_{k}$ are indeed located at $s=\Im \gamma_{n}$ [24]. In another words, the classical eigenmodes were obtained from the experimentally measured quantum spectrum for this open system. Similar experiments on closed billiards should yield results that can be compared with those discussed in the present paper. We have examined simulations of the experimental data by taking $c_{n}$ as random numbers in $[0,2 \epsilon / \pi]$ and set $\epsilon_{n}=\epsilon$, the same width for all eigenvalues as we used in $\delta \rho_{\epsilon}(k)$. We find that spectral correlations are almost identical if $c_{n}$ is constant (as in $\left.\delta \rho_{\epsilon}(k)\right)$ or uniformly distributed in $[0,2 \epsilon / \pi]$.

Classical eigenmodes play an important role in quan- 
tum dynamics. For example they govern the time evolution of a wave packet and are related to the problem of decoherence [34]. Consider the quantity

$$
A(t)=\sum a_{n} e^{-i \hbar k_{n}^{2} t / 2 \mathfrak{m}}
$$

with $\mathfrak{m}$ the particle mass, and $a_{n}$ is significant only for $K_{0}-\Delta<k_{n}<K_{0}+\Delta$ with $K_{0} \gg \Delta$. This quantity is related to the wave packet revival [35]. For two-dimensional billiards, the Heinsenberg time is $t_{H}=\mathfrak{m} A / h$. For the wave packet moving with a speed $v=\hbar K_{0} / \mathfrak{m}$, we define the Heinsenberg length $l_{H}=v t_{H}=(A / 2 \pi) K_{0}$. So one has $l_{H} \rightarrow \infty$ if $A \rightarrow \infty$ (open systems) or $K_{0} \rightarrow \infty$ (short wavelength). One has $\hbar k_{n}^{2} t / 2 \mathfrak{m}=k_{n} l-K_{0} l / 2+$ $(A / 4 \pi)\left(k_{n}-K_{0}\right)^{2}\left(l / l_{H}\right)$. Here $l=v t$. For $l \ll l_{H}$, the last term can be ignored, thus $|A(t)|^{2} \simeq\left|\sum_{n} a_{n} e^{-i k_{n} l}\right|^{2}$. In the case $a_{n}=1$, one has

$$
|A(t)|^{2} \simeq \Re \int C_{\rho_{\epsilon}}(s) e^{i s l} d s \simeq \Re \operatorname{tr} e^{\hat{L} t} .
$$

So before the Heisenberg time, $|A(t)|^{2}$ is governed directly by the classical eigenmodes $\left\{\gamma_{n}\right\}$ of the Liouville operator $\hat{L}$.

Boundary conditions play an important role on the dynamics. This was addressed recently by Biswas [36]. Non-identical boundary condition on adjacent edges of the polygonal billiards can lead not only to quantum splitting but also to quantum annihilation of $\mathrm{PO}$ families in the quantum trace formula. Such as in the case of a rectangle billiard with rational aspect ratio and only one side is with NBC. The most dramatic case is a square billiard with DBC on all sides except one. In this case, the quantum annihilation leads to a massive disappearance of PO families in the quantum trace formula. Similar to the case of quantum splitting, the spectral rigidity will also deviate from the Poissonian value due to quantum annihilation.

Previous studies of the quantum properties of billiard systems were focused on the universality of level statistics, such as nearest level spacing, spectral rigidity, etc., and the quantum-classical correspondence was typically addressed in terms of POs $[19,37]$. Here we take a different approach by examining correspondence between the classical and quantum spectra. In general, the classical eigenmodes are different from the quantum eigenmodes. Except for some special systems [27, 38, 39], there is no direct correspondence between the two spectra. For systems in which all the POs have the same Lyapunov exponent, there is a self-duality between quantum eigenvalues and the classical eigenmodes, such as the Riemann zeros. This self-duality leads to the resurgence $[6-10]$ and prophecy [6] of quantum eigenmodes in the spectral correlation. For systems such as the rectangle and equilateral triangle billiards we considered in this paper, there is an approximate self-duality between these two spectra, the quantum eigenmodes are also the classical eigenmodes. Prophecy [6] are also observed in these systems.
A general theory connecting the quantum fluctuation and the classical spectrum of the Perron-Frobenius operator has been developed [40-42] for diffusive systems. Due to the difficulty of getting the classical eigenmodes for generic systems, the focus was shifted onto the connection with random matrix theory. Since the spectral form factor is not self-averaging for clear systems, smoothing or ensemble average is required for the form factor of a single system to approach the prediction of random matrix theory [43]. Our previous study confirmed that for hyperbolic open systems, the quantum correlations are determined by the classical eigenmodes [24]. In this paper, we have confirmed this for integrable closed billiards. Thus one is able to extend the so-called AAA-BK theory $[40,41]$ to a finite spectrum of a single closed system.

\section{Acknowledgments}

We thank J.V. José for discussions. This work is supported partially by NSF-PHY-0457002.

\section{APPENDIX A: DERIVATIONS OF CLASSICAL TRACE FORMULAS}

In this appendix, we derive the trace formulas Eq. (23) and (27) from Eq. (12) and (16), respectively.

For the rectangle billiard, one has explicitly

$$
\operatorname{tr} e^{\hat{L} t}=\frac{2}{\pi} \int_{0}^{\pi / 2} \sum_{m, n=-\infty}^{\infty} e^{i m(\pi / a) l \cos \alpha+i n(\pi / b) l \sin \alpha} d \alpha .
$$

Using the identity

$$
\sum_{m=-\infty}^{\infty} e^{2 \pi i m x}=\sum_{m=-\infty}^{\infty} \delta(x-m),
$$

one gets for $l>0$

$$
\operatorname{tr} e^{\hat{L} t}=\frac{8 a b}{\pi} \sum_{m, n=-\infty}^{\infty} F_{m, n}^{\mathrm{rec}}(l)
$$

with

$$
F_{m, n}^{\mathrm{rec}}(l)=\int_{0}^{\pi / 2} \delta(l \cos \alpha-2 m a) \delta(l \sin \alpha-2 n b) d \alpha .
$$

The above defined function $F_{m, n}^{\mathrm{rec}}(l)$ is nonzero only if both $m$ and $n$ are non-negative. So we only need to consider $m, n \geq 0$. Using the property of the $\delta$-function in the polar coordinate system [44]

$$
\begin{aligned}
\delta^{2}\left(\mathbf{r}-\mathbf{r}_{0}\right) & \equiv \delta\left(x-x_{0}\right) \delta\left(y-y_{0}\right) \\
& =\frac{1}{r_{0}} \delta\left(r-r_{0}\right) \delta\left(\theta-\theta_{0}\right)
\end{aligned}
$$


we get for $m, n>0$,

$$
\begin{aligned}
F_{m, n}^{\mathrm{rec}}(l) & =\frac{1}{L_{m n}} \delta\left(l-L_{m n}\right) \int_{0}^{\pi / 2} \delta\left(\alpha-\alpha_{m n}\right) d \alpha \\
& =\frac{1}{L_{m n}} \delta\left(l-L_{m n}\right)
\end{aligned}
$$

with $L_{m n}=2 \sqrt{m^{2} a^{2}+n^{2} b^{2}}$ and $\alpha_{m n}=\tan ^{-1}(n b / m a)$. For $F_{m, 0}^{\text {rec }}(l)$ with $m>0$, since $\alpha_{m 0}=0$ and $\int_{0}^{\infty} \delta(x) d x=$ $1 / 2$, one gets $F_{m, 0}^{\mathrm{rec}}(l)=\delta\left(l-L_{m 0}\right) / 2 L_{m 0}$. Similarly one has $F_{0, n}^{\mathrm{rec}}(l)=\delta\left(l-L_{0 n}\right) / 2 L_{0 n}$ and $F_{0,0}^{\mathrm{rec}}(l)=\delta(l) / 2 l$. So one gets

$$
\begin{aligned}
\operatorname{tr} e^{\hat{L} t}= & \frac{2 a b}{\pi}\left\{\frac{2}{l} \delta(l)+2 \sum_{m=1}^{\infty}\left[\frac{\delta\left(l-L_{m 0}\right)}{L_{m 0}}+\frac{\delta\left(l-L_{0 m}\right)}{L_{0 m}}\right]\right. \\
& \left.+4 \sum_{m, n=1}^{\infty} \frac{\delta\left(l-L_{m n}\right)}{L_{m n}}\right\} .
\end{aligned}
$$

The Laplace transform of the above expression without the first term $\delta(l) / l$ will give Eq. (23).

For the circle billiard, the trace of the Liouvillian dynamics is

$$
\operatorname{tr} e^{\hat{L} t}=8 a^{2} \sum_{m, n=-\infty}^{\infty} F_{m, n}^{\mathrm{cir}}(l)
$$

with

$$
F_{m, n}^{\operatorname{cir}}(l)=\int_{0}^{\pi} d \alpha \sin ^{2} \alpha \delta\left(\frac{l}{\sin \alpha}-2 m a\right) \delta\left(\frac{\alpha l}{\sin \alpha}-2 n \pi a\right) .
$$

The above defined $F_{m, n}^{\mathrm{cir}}(l)$ will be nonzero only for $m, n \geq$ 0 . For $m>0$, using

$$
\delta\left(\frac{l}{\sin \alpha}-2 m a\right)=\frac{l}{2 m a \sqrt{4 m^{2} a^{2}-l^{2}}}\left[\delta\left(\alpha-\alpha_{m}\right)\right.
$$

$$
\left.+\delta\left(\alpha-\pi+\alpha_{m}\right)\right]
$$

with $\alpha_{m}=\arcsin (l / 2 m a)$ for $m>l / 2 a$, we get

$$
\begin{aligned}
F_{m, n}^{\mathrm{cir}}(l) & =\frac{l^{3}}{(2 m a)^{3} \sqrt{4 m^{2} a^{2}-l^{2}}} \delta\left(\alpha_{m} l \sin \alpha_{m}-2 n \pi a\right) \\
& =\frac{l^{3}}{(2 m a)^{4} \sqrt{4 m^{2} a^{2}-l^{2}}} \delta\left(\alpha_{m}-n \pi / m\right) \quad(\mathrm{A} 10)
\end{aligned}
$$

which is valid only for $m>2 n$. Since $\alpha_{m}=n \pi / m$ will give $l=L_{m n} \equiv 2 m a \sin (n \pi / m)$, thus

$$
\delta\left(\alpha_{m}-n \pi / m\right)=\sqrt{4 m^{2} a^{2}-L_{m n}^{2}} \delta\left(l-L_{m n}\right),
$$

we obtain

$$
F_{m, n}^{\mathrm{cir}}(l)=\frac{\sin ^{4}(n \pi / m)}{L_{m n}} \delta\left(l-L_{m n}\right) .
$$

One can verify that this expression is valid for all $m \geq$ $n>0$. For $m=0$ and $n \geq 0$, one has $F_{0, n}^{\text {cir }}(l)=0$ while $F_{m, 0}^{\mathrm{cir}}(l)=l^{3} \delta(l) / \pi(2 m a)^{4}$ for $m>0$. If only the contributions from $m, n>0$ are included, one gets

$$
\begin{aligned}
\operatorname{tr} e^{\hat{L} t} & =8 a^{2} \sum_{n=1}^{\infty} \sum_{m=n}^{\infty} \frac{\sin ^{4}(n \pi / m)}{L_{m n}} \delta\left(l-L_{m n}\right) \\
& =\frac{2}{\pi} \sum_{n=1}^{\infty} \sum_{m=2 n}^{\infty} \frac{a_{m n}}{L_{m n}} \delta\left(l-L_{m n}\right) .
\end{aligned}
$$

with

$$
a_{m n}=4 \pi a^{2} f_{m n} \sin ^{4}(n \pi / m)
$$

and $f_{m n}$ given in Eq. (28). A Laplace transform will give Eq. (27).
[1] D. Ruelle, Phys. Rev. Lett. 56, 405 (1986); J. Stat. Phys. 44, 281 (1986).

[2] M. Pollicott, Ann. Math. 131, 331 (1990).

[3] J. Wilkie and P. Brumer, Phys. Rev. A 55, 27; 43 (1997).

[4] D.L. Miller, Phys. Rev. E 57, 4063 (1998).

[5] D. Cohen, J. Phys. A 31, 277 (1998).

[6] W.T. Lu and S. Sridhar, arXiv: nlin.CD/0405058.

[7] M.V. Berry and J. keating, SIAM Review 41, 236 (1999).

[8] O. Bohigas, P. Leboeuf, and M. J. Sanchez, Found. Phys. 31, 489 (2001); R.D. Connors and J.P. Keating, ibid. 31, 669 (2001).

[9] P. Leboeuf, A.G. Monastra, and O. Bohigas, Regular and Chaotic Dynamics 6, 205 (2001).

[10] P. Leboeuf, Phys. Rev. E 69, 026204 (2004).

[11] H. Goldstein, Classical Mechanics, 2nd ed., AddisonWesley Publishing Company (1980), ch.10.

[12] A.M. Ozorio de Almeida, Hamiltonian Systems: Chaos and Quantization, Cambridge University Press (1988).

[13] D. Biswas, Phys. Rev. E 63, 016213 (2000); D. Biswas,
arXiv: chao-dyn/9804013.

[14] P.J. Richens and M.V. Berry, Phys. D 2, 495 (1981).

[15] K. Richter, D. Ullmo, and R.A. Jalabert, Phys. Rep. 276, 1 (1996).

[16] J.L. Vega, T. Uzer, and J. Ford, Phys. Rev. E 48, 3414 (1993).

[17] W. Bauer and G.F. Bertsch, Phys. Rev. Lett. 65, 2213 (1990).

[18] P. Cvitanovic and B. Eckhardt, J. Phys. A 24, L237 (1991).

[19] M. C. Gutzwiller, Chaos in classical and quantum mechanics, Springer-Verlag, 1990.

[20] M. Brack and R.K. Bhaduri, Semiclassical Physics, Addison-Wesley Publishing Company (1997), ch.7.

[21] E. Bogomolny, Nonlinearity 13, 947 (2000).

[22] U. Smilansky and B. Verdene, J. Phys. A 36, 3525 (2003).

[23] S.M. Reimann, M. Brack, A.G. Magner, J. Blaschke, and M.V.N. Murthy, Phys. Rev. A 53, 39 (1996).

[24] K. Pance, W.T. Lu, and S. Sridhar, Phys. Rev. Lett. 
85, 2737 (2000); W.T. Lu, K. Pance, P. Pradhan, and S. Sridhar, Phys. Scr. T90, 238 (2001).

[25] E. Gutkin, Phys. D 19, 311 (1986).

[26] M. Boshernitzan, G. Galperin, T. Krüger, and S. Troubertzkoy, Trans. Am. Math. Soc. 350, 3523 (1998).

[27] D. Biswas, Phys. Rev. Lett. 93, 204102 (2004).

[28] R. Aurich, T. Hesse, and F. Steiner, Phys. Rev. Lett. 74, 4408 (1995).

[29] W.-M. Zheng, Phys. Rev. E 60, 2845 (1999).

[30] D. Biswas, Phys. Rev. E 61, 5129 (2000).

[31] I.S. Gradshteyn and I.M. Ryzhik, Table of Integrals, Series, and Products, 5th Ed., Academic Press, 1994, (6.512.3), (6.693.2), (8.521.1).

[32] S.R. Jain, Phys. Rev. E 50, 2355 (1994).

[33] S. Sridhar, Phys. Rev. Lett. 72, 2175 (1994); H. Alt et al, Phys. Rev. E 54, 2303 (1996).
[34] W.H. Zurek, Rev. Mod. Phys. 75, 715 (2003).

[35] R.W. Robinett, Phys. Rep. 392, 1 (2004).

[36] D. Biswas, Phys. Rev. E 57, R3699 (1998).

[37] M.V. Berry, Proc. R. Soc. Lond. A 400, 229 (1985).

[38] D. Biswas and S.R. Jain, Phys. Rev. A 42, 3170 (1990).

[39] D. Biswas and S. Sinha, Phys. Rev. Lett. 71, 3790 (1993).

[40] O. Agam, B.L. Altshuler, and A.V. Andreev, Phys. Rev. Lett. 75, 4389 (1995).

[41] E. B. Bogomolny and J. Keating, Phys. Rev. Lett. 77, 1472 (1996).

[42] M.M. Sano, Phys. Rev. E 59, R3795 (1999); ibid. 66, 046211 (2002).

[43] R.E. Prange, Phys. Rev. Lett. 78, 2280 (1997).

[44] See http://mathworld.wolfram.com/DeltaFunction.html. 